\section{Avaliação da sobrecarga do cuidador de crianças com paralisia cerebral através da escala Burden Interview}

\section{Assessment of the caregiver burden of children with cerebral palsy using the Burden Interview scale}

Ana Cristina Resende Camargos 1

Tatiana Teixeira Barral de Lacerda 2

Sabrina Oliveira Viana 3

Lívia Renata Alves Pinto 4

Mara Luiza Santos Fonseca 5

1-5 Pontifícia Universidade Católica de Minas Gerais (PUC Betim) Clínica de Fisioterapia. Rua do Rosário, 1081. Betim, MG, Brasil. CEP: 32.630-000. E-mail: anacristina.camargos@terra.com.brr

\begin{abstract}
Objectives: to evaluate factors that influence the caregiver burden of children with cerebral palsy.

Methods: 56 caregivers who worked with children with cerebral palsy aged between one and fifteen years old were evaluated. To evaluate the caregiver burden the Burden Interview scale was used, along with data on the social and economic circumstances of the children's families, the severity of motor impairment, topographic diagnosis and age of child. The Mann-Whitney and Kruskal-Wallis test were used for data analysis.

Results: poorer social and economic circumstances of the children's families $(p=0.03)$ and a lower severity of motor impairment $(p=0.05)$ in the child with cerebral palsy were associated to a heavier caregiver burden. There was no significant difference in the case of topographic diagnosis $(p=0.71)$ and age of child with cerebral palsy $(p=0.35)$.

Conclusions: understanding the factors that influence the caregiver burden is one further factor that can assist care planning and intervention for this specific group.
\end{abstract}

Key words Cerebral palsy, Quality of life, Child

\section{Resumo}

Objetivos: avaliar fatores que influenciam na sobrecarga dos cuidadores de crianças com paralisia cerebral.

Métodos: foram entrevistados 56 cuidadores de crianças com paralisia cerebral com idade entre um e 15 anos. Para avaliar a sobrecarga dos cuidadores foi utilizada a escala Burden Interview e foram coletados dados sobre nivel socioeconômico da família, gravidade do comprometimento motor, diagnóstico topográfico e idade das crianças com paralisia cerebral. Para análise dos dados foram utilizados os testes Mann-Whitney e Kruskal-Wallis.

Resultados: um menor nivel socioeconômico da familia $(p=0,03)$ e uma menor gravidade do comprometimento motor $(p=0,05)$ das crianças com paralisia cerebral foram associados com maior sobrecarga do cuidador. Não houve diferença significativa em relação ao diagnóstico topográfico $(p=0,71)$ e à idade das crianças com paralisia cerebral $(p=0,35)$.

Conclusões: o conhecimento de fatores que influenciam a sobrecarga dos cuidadores de crianças com paralisia cerebral é mais um dado a ser agregado no planejamento de atenção e intervenção a esse público específico.

Palavras-chave Paralisia cerebral, Qualidade de vida, Criança 


\section{Introdução}

A paralisia cerebral (PC) é definida como um grupo de desordens do movimento e da postura que causam limitação da atividade e que são atribuídas a distúrbios não progressivos ocorridos durante o desenvolvimento do cérebro imaturo. ${ }^{1}$ A PC é considerada a incapacidade física mais comum na infância, acometendo duas crianças a cada 1000 nascimentos. ${ }^{2-4}$ A classificação da PC é baseada nos distúrbios do tônus muscular como espasticidade, discinesia, ataxia e forma mista, na distribuição anatômica do comprometimento como hemiplegia, diplegia e quadriplegia e na gravidade do quadro clínico.5-7

A PC é considerada uma condição crônica, protótipo de incapacidade na infância, que leva a limitações funcionais em longo prazo. 8 A presença de doenças crônicas não altera somente a vida dos indivíduos acometidos, mas também pode influenciar múltiplos aspectos da vida de seus cuidadores. ${ }^{9}$ Ter uma criança com PC torna-se um desafio imenso para os pais, uma vez que estes devem tentar controlar os problemas de saúde de sua criança, além de tentar manter suas atividades de vida diária. A demanda da família de crianças com PC é significativamente maior, já que essa necessita participar mais ativamente do cuidado de suas crianças.10,11 A existência de uma criança com PC altera a estrutura familiar, por aumentar a necessidade de tempo para cuidados em casa e os recursos financeiros devido aos gastos dispensados ao tratamento.

A saúde psicológica e física dos cuidadores pode ser fortemente influenciada pelo comportamento da criança e pela demanda de cuidado.12 Assim, o difícil processo de cuidar de crianças com PC, aliado ao aumento das responsabilidades que essa função promove, pode levar ao cansaço, isolamento, sobrecarga e estresse dos cuidadores. 9 Dessa forma, o cuidador primário da criança com PC, que é normalmente a mãe, altera sua vida em função de melhorar a condição de sua criança e passa a não desenvolver seus próprios papéis sociais. ${ }^{11}$

Estudos têm demonstrado que a qualidade de vida dos cuidadores de crianças com PC encontra-se diminuída nos domínios de função física, vitalidade, saúde geral e papel emocional. ${ }^{13}$ Qualidade de vida é um constructo multidimensional, subjetivo e inclui aspectos relacionados à sobrecarga do cuidador e estrutura familiar. ${ }^{9}$ Entretanto, pouco tem sido estudado sobre os fatores relacionados especificamente à sobrecarga dos cuidadores das crianças com PC.

Dessa forma, o objetivo desse estudo foi avaliar a influência do nível socioeconômico da família, da gravidade do comprometimento motor, do diagnóstico topográfico e da idade da criança com PC na sobrecarga dos cuidadores.

\section{Métodos}

Foi realizado um estudo descritivo transversal na Associação de Pais e Amigos dos ExcepcionaisBetim (APAE-Betim) e na Clinica de Fisioterapia da Pontifícia Universidade Católica de Minas Gerais (PUC) no campus de Betim, Minas Gerais, Brasil. Os critérios de inclusão foram: ser o cuidador responsável, viver com a criança e não apresentar qualquer doença crônica; e foram excluídos os cuidadores de mais de uma criança com PC ou com qualquer outra doença crônica como distrofia muscular, espinha bífida, etc. Foi utilizada uma amostra de conveniência, e todos os cuidadores de crianças atendidas nessas duas instituições que preencheram os critérios de inclusão foram convidados a parti-cipar do estudo. Os cuidadores que desejaram parti-cipar assinaram o termo de consentimento livre e esclarecido. O estudo foi aprovado pelo Comitê de Ética em Pesquisa da PUC, parecer no. CAAE 0071.0.213.000-06.

A classificação da gravidade do comprometimento motor foi realizada utilizando-se a escala Gross Motor Function Classification System (GMFCS),7,14 que foi traduzida para a língua portuguesa. 15 Apesar dessa escala não ter sido ainda validada para a população brasileira, ela tem sido utilizada em estudos neste país para classificar crianças com PC. 16 O GMFCS é um sistema de classificação que leva em conta o grau de comprometimento motor e abrange cinco níveis, graduados de I a $\mathrm{V}$, com especificações de aquisições motoras nos diferentes níveis de acordo com a idade. O nível I representa o grupo mais leve, composto pelas crianças que andam sem restrições, mas tem limitações para correr e saltar. O nível II corresponde a um grupo que anda sem auxílio de aparelhos e muletas, mas tem limitações para andar fora de casa e na comunidade. No nível III a criança consegue andar com auxílio de andadores ou muletas, mas tem dificuldades para andar fora de casa e na comunidade. O nível IV representa um grupo que anda com auxílio externo, com limitações, e necessita de cadeira de rodas para andar fora de casa e na comunidade. O nível $\mathrm{V}$ abrange o grupo de comprometimento motor mais grave, em que a criança possui uma mobilidade gravemente limitada, mesmo com aparelho e adaptações.7,14 O nível do comprometimento motor medido pelo GMFCS foi dividido em 
dois grupos, sendo o primeiro composto pelos níveis I, II e III (crianças com quadros mais leves) e o segundo pelos níveis IV e V (crianças mais graves). 16,17

Os dados em relação ao diagnóstico topográfico, sexo e idade das crianças foram coletados dos prontuários. A idade das crianças foi dividida em dois grupos, sendo o primeiro referente às crianças até sete anos (crianças mais jovens) e o segundo referente às crianças acima de sete anos (crianças mais velhas). ${ }^{17}$ Os dados relacionados à idade, ao sexo $\mathrm{e}$ ao grau de parentesco dos cuidadores com a criança foram perguntados diretamente aos mesmos por duas avaliadoras treinadas, que também foram responsáveis pela coleta de dados dos prontuários, bem como pela aplicação dos questionários aos cuidadores. Cada avaliadora ficou responsável por coletar os dados e por entrevistar metade dos cuidadores.

Para avaliar o nível socioeconômico foi utilizado o Questionário de Classificação Socioeconômica, segundo critério proposto pela Associação Brasileira de Institutos de Pesquisa de Mercado (ABIPEME). 18 As perguntas e a marcação das respostas foram feitas pelas avaliadoras. Trata-se de um instrumento que permite a categorização segundo padrões ou potencias de consumo, no qual são pontuados o grau de escolaridade do chefe da família e os itens de posse de conforto no lar. Dessa forma, classifica as camadas da população por meio de cinco classes, denominadas A, B, C, D e E, sendo a classe A o maior nível socioeconômico e a classe $\mathrm{E}$ o menor, de acordo com a pontuação recebida. As perguntas e a marcação das respostas foram feitas pelas avaliadoras. Trata-se de um instrumento que permite a categorização segundo padrões ou potenciais de consumo, no qual são pontuados o grau de escolaridade do chefe da família e os itens de posse de conforto no lar. A classificação socioeconômica (ABIPEME) foi dividida em dois grupos, sendo o primeiro composto pelas classes $\mathrm{B}$ e $\mathrm{C}$ e segundo pelas classes D e E.

Para avaliar a sobrecarga dos cuidadores foi utilizada a versão brasileira da escala Burden Interview (BI) que contém 22 perguntas englobando as áreas de saúde, vida social e pessoal, situação financeira, bem-estar emocional e relacionamento interpessoal. Essa escala pode ser utilizada para avaliar a sobrecarga dos cuidadores de indivíduos com incapacidades mental e física, 19,20 e foi recentemente traduzida e validada para a população brasileira.21 A BI foi construída tanto para ser autoaplicável quanto para ser aplicada por um entrevistador. $\mathrm{Na}$ segunda forma, o entrevistador deve ler cada item e pedir ao entrevistado para apontar a resposta correta. É aconselhável também fazer um cartão de respostas, com letras e números grandes para auxiliar a resposta do entrevistado. 21

No presente estudo, os examinadores leram as perguntas do questionário e o entrevistado respondeu conforme as opções de respostas contidas no cartão confeccionado para este fim. As duas avaliadoras responsáveis pela coleta de dados foram orientadas a realizar as perguntas através da leitura direta do questionário na íntegra, evitando assim induzir a resposta dada pelos cuidadores. Cada resposta era pontuada em uma escala do tipo Likert que varia entre 0-4 pontos. As questões referentes aos itens 1 ao 21 eram pontuadas como: 0) nunca, 1) raramente; 2) algumas vezes; 3 ) frequentemente e 4) sempre; e do item 22 como: 0) nem um pouco; 1) um pouco; 2) moderadamente; 3 ) muito e 4 ) extremamente. Todas as perguntas deveriam ser obrigatoriamente pontuadas e o escore final da BI era obtido com a soma total de todas as respostas, podendo variar de 0 a 88 . Quanto maior o escore final, maior a sobrecarga do cuidador. 21

A análise estatística foi realizada utilizando o programa SPSS versão 13.0 para Windows. Foi realizada estatística descritiva para caracterização da amostra e foi analisada a associação entre a variável resposta (escala Burden Interview) e as demais variáveis (nível socioeconômico familiar - critério ABIPEME, idade, gravidade do comprometimento motor - GMFCS e diagnóstico topográfico da criança). Os três primeiros foram divididos em dois grupos e foram analisados através do teste MannWhitney. O diagnóstico topográfico foi dividido em três grupos e analisado através do teste Kruskal Wallis. O nível de significância foi considerado quando $p<0,05$. O poder do teste $(1-\beta)$ foi de $75 \%$, baseado no tamanho da amostra utilizada neste estudo $(\mathrm{n}=56)$.

\section{Resultados}

A amostra foi composta por 56 cuidadores de crianças com PC, com idade variando entre 17 e 66 anos $(34,75 \pm 10,4)$, sendo 54 mulheres e dois homens. Do total de participantes, 45 eram mães, dois eram pais, seis eram avós, uma era tia e duas eram primas. A renda familiar dos participantes variou de zero a onze e meio salários mínimos. Em relação ao nível socioeconômico, quatro participantes foram categorizados na classe B, 24 na classe C, 22 na classe $\mathrm{D}$ e seis na classe $\mathrm{C}$.

$\mathrm{Na}$ avaliação da sobrecarga dos cuidadores 
Tabela 1

Resultados da análise bivariada entre as variáveis independentes e o escore obtido na escala Burden Interview em cuidadores de crianças com paralisia cerebral.

\begin{tabular}{|c|c|c|c|c|}
\hline \multirow[t]{2}{*}{ Variáveis independentes } & \multicolumn{4}{|c|}{ Variável dependente (Sobrecarga do Cuidador)* } \\
\hline & $\mathrm{n}$ & Mediana & Mínimo - Máximo & $p$ \\
\hline \multicolumn{5}{|c|}{ Nível socioeconômico (ABIPEME)** } \\
\hline Classes B e C & 28 & 16,5 & $6-36$ & 0,03 \\
\hline Classes D e E & 28 & 23,0 & $4-48$ & --- \\
\hline \multicolumn{5}{|l|}{ Idade } \\
\hline Até 7 anos & 42 & 21,0 & $4-42$ & 0,35 \\
\hline Acima de 7 anos & 14 & 22,5 & $11-48$ & --- \\
\hline \multicolumn{5}{|c|}{ Comprometimento motor (GMFCS)*** } \\
\hline Nível I, II e III & 23 & 27,0 & $4-48$ & 0,049 \\
\hline Níveis IV e V & 33 & 19,0 & $6-42$ & --- \\
\hline \multicolumn{5}{|l|}{ Diagnóstico topográfico } \\
\hline Quadriplegia & 27 & 20,0 & $6-48$ & 0,71 \\
\hline Diplegia & 17 & 23,0 & $4-38$ & --- \\
\hline Hemiplegia & 12 & 23,0 & $6-41$ & --- \\
\hline
\end{tabular}

* Avaliada pela escala Burden Interview (Scazufca, 2002); ** Questionário de Classificação Socioeconômica (ABIPEME, 2003) ; *** Sistema de Classificação da Função Motora Grossa (Palisano et al.14).

através da Escala BI foi encontrado um escore mínimo de quatro e máximo de $48(21,95 \pm 10,16)$.

A idade das crianças variou entre 1 e 15 anos $(5,6 \pm 3,6)$. Dessas, 15 eram quadriplégicas espásticas, 15 diplégicas espásticas, 12 hemiplégicas espásticas, três atetóides, uma atáxica e 10 eram do tipo misto.

Em relação ao GMFCS, dezesseis crianças pertenciam ao nível I, cinco pertenciam ao nível II, dois pertenciam ao nível III, sete pertenciam ao nível IV e vinte e seis pertenciam ao nível V.

Houve diferença significativa entre os dois grupos de classe socioeconômica e a sobrecarga do cuidador $(p=0,03)$, sendo a mediana da sobrecarga do cuidador da categoria $\mathrm{B}$ e $\mathrm{C}$ inferior às categorias D e E (Tabela 1).

A diferença foi significativa entre os grupos de comprometimento motor e a sobrecarga do cuidador ( $p=0,049)$, sendo que a mediana da sobrecarga do cuidador das crianças com quadros mais leves foi maior quando comparado às crianças mais graves (Tabela 1).

O diagnóstico topográfico das crianças foi dividido em três grupos: quadriplegia, diplegia e hemiplegia (Tabela 1). Não houve diferença significativa entre os grupos do diagnóstico topográfico e a sobrecarga do cuidador $(p=0,71)$.

Não houve diferença significativa entre os grupos de idade em relação à sobrecarga do cuidador $(p=0,35)$ (Tabela 1).

\section{Discussão}

As crianças com PC constituem um grupo de pacientes com prejuízo motor que se manifesta principalmente nas atividades de vida diária e de vida prática. Essas alterações podem gerar grandes repercussões na realização de tarefas simples e corriqueiras, afetando a rotina das próprias crianças e de seus familiares. $8,11,12$ Este estudo procurou identificar possíveis fatores que poderiam estar relacionados com a sobrecarga dos cuidadores de crianças com PC.

No presente estudo, houve diferença significativa entre os dois grupos de nível socioeconômico segundo critério ABIPEME (classes B e C / D e E), sendo encontrada maior sobrecarga dos cuidadores em famílias com condições socioeconômicas menos favoráveis. Esses achados coincidem com os de Friedrich et al.,22 que demonstraram que os cuidadores de crianças com incapacidade com nível socioeconômico elevado controlam melhor o seu bem-estar quando comparados com cuidadores com nível socioeconômico inferior. Segundo Ute et al., ${ }^{23}$ um terço das mães de crianças com doenças crônicas necessitam parar de trabalhar para cuidar de seus filhos que estão em tratamento. Além disso, cuidar de uma criança com incapacidade aumenta a demanda de recursos, incluindo tempo e dinheiro, 10 o que pode aumentar a sobrecarga dos cuidadores, quando esses são membros economicamente ativos 
da família.

No presente estudo, a sobrecarga dos cuidadores de crianças com comprometimentos motores mais leves foi maior do que a de crianças mais graves. Uma provável explicação pode ser o fato de que crianças com comprometimentos mais leves apresentam maior independência e participação na vida social. De acordo com Mancini et al. 24 e Silva et al., ${ }^{25}$ um menor nível do comprometimento motor parece ser indicativo de maior participação escolar da criança. Entretanto, essas crianças que frequentam escolas regulares podem apresentar dificuldades em atender às expectativas devido aos prejuízos motores apresentados, o que afeta sua habilidade para participar ativamente nas atividades de aprendizado escolar, recreação e atividade física, expressar seus anseios e/ou interagir com os colegas. 26 Assim, é possível inferir que esses fatores podem gerar frustração e angústia dos familiares e gerar um aumento da sobrecarga emocional dos mesmos.

Entretanto, no estudo de Tuna et al.13 não foi encontrada diferença entre a gravidade do comprometimento motor e a qualidade de vida dos cuidadores de crianças com PC mensurada pelo questionário Short-Form (SF-36). A escala BI avalia questões relacionadas à saúde, vida pessoal e social, situação financeira, bem-estar emocional e relacionamento inter-pessoal.20 Já o SF-36 avalia questões relacionadas à função física, dor corporal, vitalidade, saúde geral, função social e limitações sociais relacionadas aos problemas físicos e emocionais. 13 Esses achados foram contrastantes provavelmente devido às diferenças de constructo das medidas de desfecho.

No presente estudo não houve diferença significativa na sobrecarga do cuidador em relação ao diagnóstico topográfico das crianças com PC. Nos casos pesquisados nesse trabalho não foi encontrada relação entre essa variável e a sobrecarga dos cuidadores. A literatura apenas ressalta a relação do diagnóstico topográfico com a qualidade de vida das próprias crianças. 27 No estudo de Bjornson e McLaughlin, 27 as crianças com diagnóstico topográfico de quadriplegia espástica obtiveram um escore inferior no questionário aplicado sobre saúde da criança, quando comparadas às crianças com diagnóstico topográfico de diplegia ou hemiplegia espástica. Segundo Vargus-Adams, 28 o nível de comprometimento motor pode interferir na habilidade das crianças em realizarem suas atividades de vida diária, interferindo, portanto, na qualidade de vida das mesmas. Não se pode afirmar, desta forma, que haja uma relação linear entre a qualidade de vida das crianças e de seus cuidadores.

Outra variável pesquisada foi a idade das crianças com PC que, para análise, foi dividida em dois grupos: crianças mais jovens (até os sete anos) e crianças mais velhas (acima de sete anos). De acordo com Palisano et al., 14 com sete anos de idade as crianças com PC iniciam um estágio de platô em relação à aquisição das habilidades e podem até mesmo perder habilidades adquiridas. Knox e Evans 17 encontraram que crianças até os sete anos de idade apresentam melhora significativa durante programas de reabilitação em relação às crianças acima de sete anos. Apesar de serem observadas tais diferenças na evolução do quadro clínico de crianças com PC, no presente estudo não foi identificada diferença em relação à sobrecarga do cuidador entre os dois grupos de idade.

Além disso, pode-se constatar que a variável resposta (Escala BI) apresentou pequena variabilidade entre os dados. O escore máximo da escala é de 88 pontos e indica maior sobrecarga do cuidador. 21 Neste estudo, o escore variou somente de 4 a 48 , o que não indica uma sobrecarga muita elevada dos cuidadores na amostra estudada. De acordo com Raina et al.,12 o suporte social auxilia nas estratégias de enfrentamento e pode influenciar positivamente na percepção da sobrecarga. Por outro lado, não se pode descartar a possibilidade de o escore ter sido baixo porque os entrevistados ficaram com algum receio, ou até mesmo com vergonha de responder honestamente sobre sua relação com a tarefa de cuidar da criança. A forma de aplicação da Escala BI escolhida nesse trabalho pode ter influenciado nos resultados, pois o entrevistador precisou ler as perguntas para os cuidadores, uma vez que alguns indivíduos da população em questão não eram alfabetizados. Além do grau de instrução do cuidador, outros fatores também podem influenciar a sobrecarga dos cuidadores, como o estado civil, a religiosidade, a divisão do ato de cuidar com outras pessoas da família e o suporte oferecido pelas instituições que os acolhem, mas isso não foi objeto de investigação no presente estudo.

Os resultados obtidos nesse estudo não podem ser generalizados uma vez que a amostra estudada foi pequena e restrita a cuidadores de crianças com $\mathrm{PC}$ que frequentam programas de reabilitação. $\mathrm{O}$ suporte social dado por instituições de apoio, como no caso da APAE, é importante para auxiliar a família a enfrentar a situação de uma forma mais tranquila. Assim, o convívio com outras famílias que enfrentam o mesmo problema ajuda a diminuir o sofrimento e a sensação de abandono. Além disso, todos os cuidadores avaliados eram parentes e, 
muitas vezes, essa função não é considerada um encargo para muitos deles. Para Raina et al. ${ }^{12}$ cuidar é uma tarefa normal para os pais já que, na maior parte dos casos, eles se adaptam bem aos desafios de cuidar de uma criança com paralisia cerebral.

No presente estudo, os achados evidenciaram maior sobrecarga entre os cuidadores de crianças com paralisia cerebral com menor gravidade do comprometimento motor e com condições socioeconômicas desfavoráveis. Fatores como idade e

\section{Referências}

1. Bax M, Goldstein M, Rosenbaum P, Leviton A, Paneth N, Ban B, Jacobsson B, Damiano D, Executive Committee for the Definition of Cerebral Palsy. Proposed definition and classification of cerebral palsy, April 2005. Dev Med Child Neurol. 2005; 47: 571-6.

2. Stanley FJ, Blair E, Alberman E. Cerebral palsies: epidemiology and causal pathways. London: Mac Keith Press; 2000.

3. Rosen MG, Dickinson JC. The incidence of cerebral palsy. Am J Obstet Oncol. 1992; 167: 423.

4. Dzienknowski RC, Smith KK, Dillow K.A, Yucha CB. Cerebral palsy: a comprehensive review. Nurse Pract. 1996; 21: 45-8.

5. Ingram TTS. A historical review of the definition and classification of the cerebral palsies. In: Stanley FJ, Alberman ED. The epidemiology of the cerebral palsies. Spastics international. Oxford: Blackwell Scientific; 1984. p. 1-11.

6. Bleck EE. Orthopaedic management in cerebral palsy. Philadelphia: JB Lippincott; 1987.

7. Palisano RJ, Hanna SE, Rosenbaum PL, Russell DJ, Walter SD, Wood EP, Raina PS, Galuppi BE. Validation of a model of gross motor function for children with cerebral palsy. Phys Ther. 2000; 80: 974-85.

8. Raina P, O’Donnell M, Schwellnus H, Rosenbaum P, King G, Brehaut J, Russell D, Swinton M, King S, Wong M, Walter SD, Wood E. Caregiving process and caregiver burden: conceptual models to guide research and practice. BMC Pediatr. 2004; 4: 1-13.

9. Lim J, Zebrack B. Caring for family members with chronic physical illness: a critical review of caregiver literature. Health Qual Life Outcomes. 2004; 2: 1-9.

10. Brehaut JC, Kohen DE, Raina P, Walter SD, Russell DJ, Swinton M, O'Donnell M, Rosenbaum P. The health of primary caregivers of children with cerebral palsy: how does it compare with that of other Canadian caregivers? Pediatrics. 2004; 114: 182-91.

11. Ones K, Yilmaz E, Cetinkaya B, Caglar N. Assessment of the quality of life of mothers of children with cerebral palsy (primary caregivers). Neurorehabil Neural Repair. 2005; 19: 232-7.

12. Raina P, O’Donnell M, Rosenbaum P, Brehaut J, Walter SD, Russell D, Swinton M, Zhu B, Wood E. The health and well-being of caregivers of children with cerebral palsy. Pediatrics. 2005; 115: 626-36. diagnóstico topográfico das crianças não interferiram na sobrecarga dos cuidadores.

$\mathrm{O}$ conhecimento de fatores que influenciam a sobrecarga dos cuidadores com paralisia cerebral é mais um dado a ser agregado no planejamento de atenção e intervenção a esse público específico. Sugere-se que tais dados possam ser úteis para estratégias de proteção, vigilância e assistência às crianças com incapacidades.

13. Tuna H, Unalan H, Tuna F, Kokino S. Quality of life of primary caregivers of children with cerebral palsy: a controlled study with short from-36 questionnaire. Dev Med Child Neurol. 2004; 46: 646-8.

14. Palisano R, Rosenbaum P, Walter S, Russell D, Wood E, Galuppi B. Developmental and reliability of a system to classify gross motor function in children with cerebral palsy. Dev Med Child Neurol. 1997; 39: 214-23.

15. Hiratuka E. Sistema de classificação da função motora grossa para paralisia cerebral (GMFCS). [documento online]. [acesso em: $15 \mathrm{dez}$. 2006]. Disponível em: http://www.canchild.ca/Portals/0/outcomes/pdf/GMFCS _portuguese.pdf

16. Cury VCR, Mancini MC, Melo AP, Fonseca ST, Sampaio RF, Tirado MGA. Efeitos do uso de órtese na mobilidade funcional de crianças com paralisia cerebral. Rev Bras Fisioter. 2006; 10: 67-74

17. Knox V, Evans AL. Evaluation of the functional effects of a course of Bobath therapy in children with cerebral palsy: a preliminary study. Dev Med Child Neurol. 2002; 44: 44760 .

18. ABIPEME (Associação Brasileira de Institutos de Pesquisa e Mercado). Classificação socioeconômica: critério ABIPEME.2003 [documento online]. [acesso em: 26 jul. 2006]. Disponível em: http://www.ufrn.br/sites/fonaprace/ perfil_anexo3.doc.

19. Morimoto T, Schreiner AS, Asano H. Caregiver burden and health-related quality of life among Japanese stroke caregivers. Age Ageing. 2003; 32: 218-23.

20. Carod-Artal FJ, Egido-Navarro JA, González-Gutiérrez JL, Varela de Seijas E. Perception of long term overload in caregivers of patients who have survived a stroke. Rev Neurol. 1999; 28: 1130-8.

21. Scazufca M. Brazilian version of the Burden interview scale for the assessment of burden of care in carers of people with mental illnesses. Rev Bras Psiquiatr. 2002; 24 : 12-7.

22. Friedrich WN, Wilturner LT, Cohen DS. Coping resources and parenting mentally retarded children. Am J Ment Def. 1985; 90: 130-9.

23. Ute T, Kuhlthau K, Perrin JM. Employment, child care, and mental health of mothers caring for children assisted by technologt. Pediatrics. 1999; 103: 1235-42. 
24. Mancini MC, Coster WJ, Trombly CA, Heeren TC. Predicting elementary school participation in children with disabilities. Arch Phys Med Rehabil. 2000; 81: 339-47.

25. Silva AIT, Silva DBR, Agnelli LB, Higuchi MA, Oliveira MC, Silva PC, Mancini MC, Varela RCB. Perfil funcional de crianças com paralisia cerebral na escola regular segundo tipo de escola e comprometimento motor. Temas Desenv. 2004; 13: 5-13.
26. Batista MW, Enumo SRF. Inclusão escolar e deficiência mental: análise da interação social entre companheiros. Est Psicol. 2004; 9: 101-11

27. Bjornson KF, Mclaughlin JF. The measurement of healthrelated quality of life (HRQL) in children with cerebral palsy. Eur J Neurol. 2001; 8: 183-93.

28. Vargus-Adams J. Health-related quality of life in childhood cerebral palsy. Arch Phys Med Rehabil. 2005; 86: 940-5.

Recebido em 9 de maio de 2007

Versão final apresentada em 11 de dezembro de 2008

Aprovado em 19 de dezembro de 2008 